\title{
ATENÇÃO DOMICILIAR EM SAÚDE BUCAL: EXPERIÊNCIA DE INTEGRAÇÃO ENSINO-SERVIÇO-COMUNIDADE EM CENTRO DE SAÚDE DA FAMÍLIA
}

HOME CARE IN ORAL HEALTH: TEACHING-SERVICE-COMMUNITY INTEGRATION EXPERIENCE AT A FAMILY HEALTH CENTER

ATENCIÓN DOMICILIARIA EN SALUd BUCAL: EXPERIENCIA DE INTEGRACIÓN ENSEÑANZA-SERVICIO-COMUNIDAD EN UN CENTRO DE SALUD DE LA FAMILIA

André Pereira de Lima ${ }^{1}$

Timóteo Sousa Lopes 2

Atanara Freires Aguiar de Lima ${ }^{3}$

Mariana Ramalho de Farias ${ }^{4}$

Jacques Antonio Cavalcante Maciel ${ }^{5}$

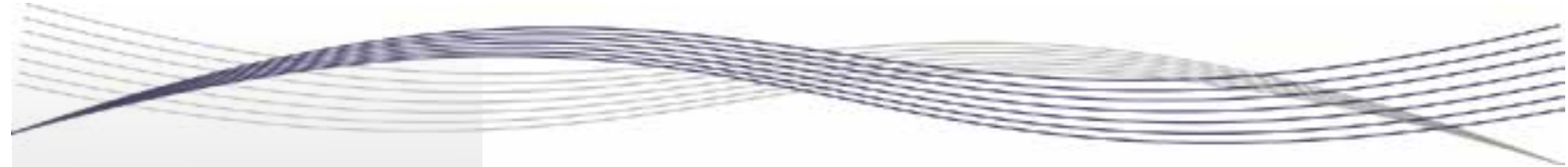

Palavras-chave:

Visita Domiciliar; Saúde Bucal; Atenção Primária à Saúde.

Keywords: Home Visit; Oral Health; Primary Health Care.

Palabras clave: Visita Domiciliaria; Salud Bucal; Atención Primaria de Salud.

Submetido: 28/12/2018

Aprovado: 21/04/2019

Autor(a) para Correspondência: Jacques Antonio Cavalcante Maciel

R. Lourenço Guimarães, 250

Paulo Ximenes do Prado Groairas (CE) - CEP: 62190-000

E-mail: jacques.maciel@sobral. ufc.br

\section{RESUMO}

Este estudo objetivou estabelecer um protocolo de atenção domiciliar (AD) em saúde bucal para pacientes restritos ao lar. Trata-se de relato de experiência baseado em uma ação de intervenção realizada por alunos de Graduação em Odontologia em Sobral-CE. Nesse contexto, mostrou-se necessária a criação do protocolo para a realização de visitas e a proposição de planos de tratamento, visando à prevenção de agravos e à promoção da saúde. A elaboração do instrumento buscou facilitar a abordagem durante as visitas e estratificar as prioridades de atenção aos pacientes restritos ao lar. 0 protocolo aborda aspectos de saúde geral relativos à saúde bucal e informações sobre o território de abrangência da equipe de saúde bucal (ESB), bem como o estabelecimento de um plano de tratamento integral. Este estudo evidenciou que a $A D$ em saúde bucal contribui para minimizar iniquidades em saúde bucal decorrentes da dificuldade de acesso aos serviços de saúde. A curto e longo prazos, espera-se garantir a continuidade das visitas, a realização das intervenções que cada paciente necessita e a diminuição da demanda de procedimentos curativistas.

1. Cirurgião-Dentista graduado pela Universidade Federal do Ceará (UFC). Sobral (CE), Brasil. E-mail: Lima. andre2011@gmail.com

2. Cirurgião-Dentista graduado pela UFC. Sobral (CE),Brasil.E-mail: timoteo.sous@hotmail.com

3. Enfermeira graduada pela Universidade Estadual Vale do Acaraú (UVA). Sobral (CE), Brasil. E-mail: atanara_ aguiar@hotmail.com

4. Cirurgiã-Dentista. Professora no Curso de Odontologia da UFC. Sobral (CE), Brasil. E-mail: marifarias_ odonto@yahoo.com.br

5. Cirurgião-Dentista. Professor no Curso de Odontologia da UFC. Groaíras (CE), Brasil. E-mail: jacques.maciel@ sobral.ufc.br 


\section{ABSTRACT}

This study aimed to establish a home care (HC) protocol in oral health for home-restricted patients. This is an experience report based on an intervention action carried out by undergraduate Dentistry students in Sobral, Ceará, Brazil. In this context, it was necessary to create a protocol for visits and treatment plan proposals, aiming at the prevention of illnesses and the promotion of health. The instrument's design sought to facilitate the approach during visits and to stratify the priorities of care for home-restricted patients. The protocol addresses general health aspects related to oral health and information about the territory covered by the oral health team $(\mathrm{OHT})$, as well as the establishment of a comprehensive treatment plan. This study showed that HC in oral health contributes to minimize inequities in oral health due to difficulty of access to health services. In the short and long terms, it is expected to guarantee the continuity of visits, the accomplishment of interventions that each patient needs, and the decreased need for cure-driven procedures.

\section{RESUMEN}

Este estudio tuvo como objetivo establecer un protocolo de atención domiciliaria (AD) en salud bucal para pacientes restringidos al hogar. Este es un informe de experiencia basado en una acción de intervención realizada por estudiantes de Pregrado en Odontología en Sobral, Ceará, Brasil. En este contexto, se mostró necesaria la creación del protocolo para visitas y la proposición de planes de tratamiento, con miras a la prevención de agravios y la promoción de la salud. La elaboración del instrumento buscó facilitar el abordaje durante las visitas y estratificar las prioridades de cuidado a los pacientes restringidos al hogar. El protocolo aborda aspectos de salud general relacionados con la salud bucal e información acerca del territorio cubierto por el equipo de salud oral (ESO), así como el establecimiento de un plan de tratamiento integral. Este estudio demostró que la AD en salud bucal contribuye a minimizar inequidades en salud bucal debido a la dificultad de acceso a los servicios de salud. A corto y largo plazo, se espera que garantice la continuidad de las visitas, el cumplimiento de las intervenciones que cada paciente necesita y menor necesidad de procedimientos curativistas.

\section{INTRODUÇÃ O}

No Sistema Único de Saúde (SUS), a organização da Estratégia Saúde da Família (ESF) em equipes multiprofissionais tem sido o pilar de um modo de pensar e praticar saúde distinto do modelo tradicional, pautado pelos princípios da atenção primária à saúde (APS). Inicialmente implementado como Programa Saúde da Família (PSF), o Brasil recorreu à ESF como opção preferencial para a APS, tendo como desafio estabelecer as inovadores diretrizes do SUS ${ }^{1}$.

A ESF prevê a atenção domiciliar (AD) como forma de assistência aos usuários que demandam cuidados contínuos, sobretudo como instrumento de diagnóstico local e programação de ações a partir da realidade, com ênfase na redução de desigualdades na atenção à saúde. A AD se caracteriza por ações sistematizadas, articuladas e regulares e pela integralidade das ações de promoção, recuperação e reabilitação da saúde e, além disso, seu principal objetivo é assistir ao paciente restrito ao lar, visando a potencializar sua autonomia e ampliar o leque de possibilidades de intervenção em domicílio com base em planejamento adequado para cada situaçã $0^{2}$.
A AD tem a visita domiciliar (VD) como um dos principais mecanismos para estabelecer vínculos com a população e seu caráter estratégico voltado à integralidade e humanização das ações de saúde traz maior proximidade e, consequentemente, corresponsabilização dos profissionais da saúde diante das demandas da população, em seu contexto social e familiar ${ }^{2}$. A AD voltada à saúde bucal na ESF qualifica a promoção da saúde bucal e a prevenção de doenças advindas da cavidade oral, além de possibilitar a realização de procedimentos odontológicos no ambiente domiciliar e de oferecer um cuidado que amplia a autonomia por meio da integração paciente-cuidador ${ }^{2,3}$.

No contexto da $A D$, os cuidados domiciliares em saúde bucal são definidos como o conjunto de ações de educação em saúde, com orientações sobre autocuidado, prevenção de agravos e assistência odontológica em domicílio. Diante do desafio de proporcionar cuidados domiciliares em saúde bucal, deve-se considerar que, na maioria das vezes, esse tipo de assistência exige equipamentos e instrumentos e impõe condições de biossegurança e de ergonomia que dificilmente são plenamente viáveis em domicílio. Com tais dificuldades em vista, 
o consultório odontológico dos serviços de saúde é o espaço privilegiado para as atividades clínicas a assistência odontológica em domicílio deve ficar restrita a casos específicos nos quais a equipe que acompanha o paciente julgar necessário, priorizando as necessidades do usuário do serviço de saúde ${ }^{4}$.

A Política Nacional de Atenção Básica (PNAB) estabelece como uma das responsabilidades comuns a todos os profissionais da ESF a realização de $A D$ voltada a pessoas com problemas de saúde controlados/compensados, com algum grau de dependência para as atividades da vida diária, que não podem deslocar-se até a unidade básica de saúde (UBS). Essa estratégia é considerada promotora de acesso às políticas públicas, mediante relação que se estabelece entre os diversos sujeitos do processo, constituindo um dos instrumentos fundamentais para a atenção integral ${ }^{5}$. Por outro lado, as diretrizes da Política Nacional de Saúde Bucal (PNSB) indicam as visitas domiciliares como um componente mais relacionado às ações educativas e preventivas, como um procedimento realizado preferencialmente pelo agente comunitário de saúde $(A C S)^{5}$.

Embora configurem práticas essenciais na APS, poucos estudos buscam a identificação e a caracterização da VD e dos cuidados domiciliares em saúde bucal no âmbito da ESF. Um desses raros estudos sobre a temática evidenciou a preocupação de que, em geral, os profissionais da equipe de saúde bucal (ESB) ainda reproduzem o modelo assistencial curativo, clínico, individualizado, pautado por tecnologias duras, enfrentando entraves para mudar tal modelo com a incorporação de práticas de prevenção de agravos e promoção da saúde e recorrendo à VD com pouca frequência ${ }^{5}$.

$\mathrm{Na}$ prática, observa-se carência de diretrizes e protocolos para a $A D$ voltada à saúde bucal. A partir dessa contextualização, constatou-se a necessidade da criação de um protocolo de AD em saúde bucal,

\section{Essa estratégia \\ é considerada promotora de acesso às políticas públicas...}

a partir da realização de VD pela ESB, visando à prevenção de agravos e promoção da saúde, bem como à redução no número de intervenções curativistas.

0 estudo objetivou estabelecer um protocolo de $A D$ em saúde bucal para pacientes restritos ao lar; assim, este artigo relata a experiência baseada em uma ação de intervenção realizada por alunos de Graduação em Odontologia em Sobral-CE.

\section{METODOLOGIA}

Trata-se de relato de experiência baseado na vivência de estágio supervisionado de alunos de Graduação em Odontologia da Universidade Federal do Ceará (UFC), realizado em Sobral no período de fevereiro a junho de 2018. Esse estágio contou com a participação de profissionais dos serviços de saúde e a supervisão de professores da UFC.

Sobral, município localizado no norte do Ceará, constitui um cenário de práticas que integra serviços de saúde e instituições de ensino - o curso de Graduação em Odontologia da UFC propicia, via atividades curriculares do “Estágio em Atenção Primária", a elaboração e execução de projetos de intervenção que busquem aprimorar a atuação da ESB no território de abrangência da ESF.

A experiência deste estudo parte de um problema observado nas primeiras visitas e por meio de relatos dos ACS e dos cirurgiões-dentistas do Centro de Saúde da Família (CSF) “Dr. Grijalba Mendes Carneiro" - conhecido como CSF Coelce -, que relataram a existência de considerável quantidade de pacientes restritos ao lar com necessidade de acompanhamento. A partir de uma primeira VD solicitada por familiares de um paciente, dos relatos da ESB e da observação dessa realidade, constatou-se a realização de poucas VDs pela ESB. Estas, em geral, ocorriam apenas quando solicitadas pelas famílias de pacientes acamados - principalmente em casos de dor. 0s ACS relataram, ainda, que muitos pacientes restritos ao lar no território coberto pelo CSF Coelce demandam maior acompanhamento por parte da ESB, mas que esta não dispunha de recursos para priorizar a quantidade estimada de casos.

\section{RESULTADOS}

Para otimizar as ações em saúde bucal junto a essa população, planejou-se a elaboração de um protocolo baseado no prontuário clínico utilizado pela Clínica Odontológica da UFC no Campus Sobral. 
Foram realizadas adaptações no protocolo para uso em ambiente domiciliar, com informações de saúde relativas às demandas dos pacientes restritos ao lar obtidas a partir das visitas; elas estratificaram a prioridade e viabilidade de assistência clínica domiciliar.

Participaram da elaboração desse instrumento: a) os alunos de Graduação em Odontologia da UFC; b) a ESB; e c) os ACS. Em um primeiro momento, realizou-se uma reunião com todos os envolvidos para elaborar perguntas que deveriam constar no protocolo e para agendar visitas a alguns dos pacientes restritos ao lar (com o propósito de ouvir o que tanto os pacientes como os cuidadores têm a dizer sobre a necessidade de tais visitas).

Em seguida, a equipe realizou visitas no território coberto pelo CSF Coelce, ouvindo pacientes, cuidadores e familiares e tomando conhecimento de algumas das necessidades desses usuários do serviço de saúde, como orientações ao cuidador sobre a saúde bucal do paciente. Uma segunda reunião foi realizada entre a ESB e os ACS para analisar as visitas realizadas e verificar a real necessidade da implementação do protocolo.

Essa intervenção se desenvolveu nos domicílios de pacientes restritos ao Lar adscritos ao CSF Coelce no início do primeiro semestre de 2018. Antes mesmo da elaboração e padronização do instrumento, as seguintes ações ocorreram nos domicílios: a) busca ativa de lesões e alterações de tecidos bucais na cavidade oral (incluindo o autoexame da cavidade oral); b) vigilância e educação em saúde bucal, com orientações sobre medidas de higiene oral (incluindo modos de escovação dentária e desinfecção de prótese); e c) encaminhamento para a atenção especializada, com participação ativa do cirurgiãodentista que atua no território.

A partir desse contato inicial, em sua composição consolidada, o protocolo aborda aspectos de saúde geral, com informações que abrangem: a) os dados pessoais; b) o território de abrangência da ESF; e c) o perfil da saúde bucal: i) histórico de assistência odontológica; ii) condições de saúde bucal; iii) possibilidades de intervenção clínica em domicílio por parte da ESB; iv) necessidade de ações de educação em saúde (coletivas ou voltadas ao paciente e/ou cuidador); v) necessidade de intervenção por parte de outros profissionais da ESF; e vi) necessidade de encaminhamento para um serviço de atenção secundária (Figura 1 ).

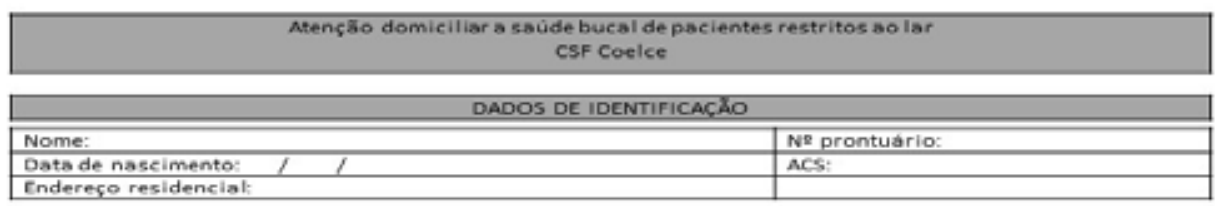

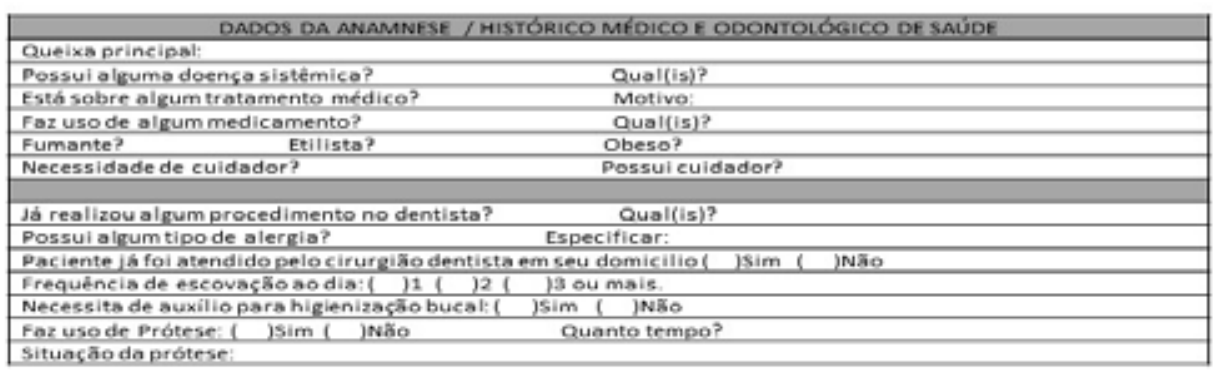
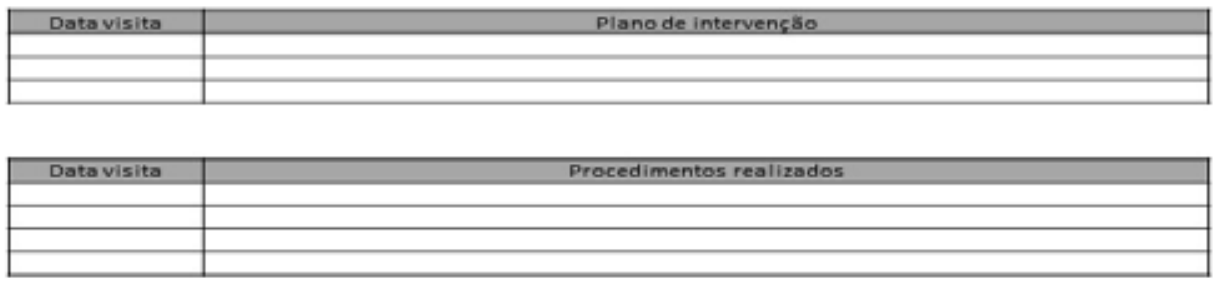

OBS:

Figura 1. Ficha utilizada nas visitas domiciliares. Sobral, 2018.

Fonte: Elaborada pelos autores. 
No período de intervenção, 16 pacientes foram examinados por meio de VD com uso desse protocolo entre esses usuários havia crianças, adultos e idosos, sendo este último o grupo prevalente de pacientes restritos ao lar. Todos os idosos se submeteram a exame clínico para prevenção do câncer de boca, com busca de alterações potencialmente malignas e das demais patologias que pudessem demandar encaminhamento para a atenção especializada. Além disso, os usuários que faziam uso de prótese receberam orientações acerca da higiene delas.

0 protocolo possibilita a elaboração de um planejamento que objetiva intervenções integrais, para melhoria da saúde bucal e global do paciente baseada em dados individuais e relacionadas ao contexto do paciente, realizadas em caráter individual ou coletivo.

Durante a experiência das visitas domiciliares, observou-se que as condições odontológicas estão diretamente relacionadas às condições socioeconômicas, ambientais, psicológicas e nutricionais. Piores condições socioeconômicas se relacionam a baixo nivel de escolaridade do paciente e/ou cuidador e esse contexto influencia o grau de compreensão da importância de manter a saúde bucal. Tais condições acabam repercutindo nas condições de moradia, já que, para a população idosa e/ou portadora de alguma deficiência física ou mental, uma casa segura, com corrimões, barras de segurança, escadas adequadas, boa iluminação, entre outros recursos, contribui para evitar acidentes domésticos que, muitas vezes, envolvem graves riscos aos moradores ${ }^{6-10}$.

0 cirurgião-dentista, enquanto profissional da saúde, assume papel fundamental na VD mediante atividades de promoção da saúde, motivação e educação em saúde voltada ao paciente e à sua família. Ele enfoca a proteção e prevenção em saúde bucal, com orientações acerca de higiene bucal e cuidados com prótese, aplicação tópica de flúor e escovação supervisionada, além de tratamento clínico após a identificação de lesões orais potencialmente malignas. Esse profissional estabelece uma rede de comunicação participativa com a família e com os demais profissionais da ESF, coordenando o cuidado do paciente restrito ao lar $^{6}$.

Durante o período de vivência, observou-se que a maioria dos pacientes restritos ao lar estava acamada no momento da visita, com limitação de movimentos e da capacidade funcional que gera empecilhos ao deslocamento para a realização de

\section{Esse profissional estabelece uma rede de comunicação participativa com a família e com os demais profissionais $d a$ ESF...}

suas atividades de vida diária ${ }^{11}$. Esse indivíduo lida com alguma fragilidade decorrente de doenças crônicas ou de outras patologias que ameaçam sua integridade física, social e econômica, suscitando situações que demandam a presença de outrem por longos períodos ${ }^{12}$.

Essas impressões relativas à dificuldade de acesso ao serviço de saúde também podem ser decorrentes de fatores como falta de meio de transporte, precária malha viária urbana, violência urbana e tráfico de drogas, o que pode aumentar ainda mais a quantidade de pacientes com restrição ao $\operatorname{lar}^{6}$. Um estudo sobre o processo de AD nas unidades da ESF que aderiram ao Programa Nacional de Melhoria do Acesso e da Qualidade da Atenção Básica (PMAQ-AB) constatou que, na prática, o cuidado em domicílio é oferecido por cerca de $50 \%$ dos profissionais das ESBs, o que evidencia que estas precisam superar desafios para implementar novas práticas de $A D$ no âmbito da APS 5 .

Ações de $A D$ no território priorizam a minimização de iniquidades resultantes de uma atenção à saúde desigual, cuja origem é a dificuldade de acesso aos serviços. 0 acesso se mostra um conceito complexo, relacionado tanto ao ato de ingressar, entrar, quanto ao termo acessibilidade, que corresponde à capacidade da população usar os recursos disponiveis. Assim, a acessibilidade é um fator da oferta para explicar as variações no uso de serviços por grupos populacionais, representando uma dimensão relevante em estudos sobre a equidade nos sistemas de saúde?

0 itinerário do território de abrangência da ESF assume vital importância na avaliação da equidade de acesso dos pacientes restritos ao lar, valendose de fatores individuais (in)capacitantes para analisar o uso de serviços de saúde. A presença de outros preditores de uso, além das demandas de saúde e dos aspectos demográficos, denota uma situação de iniquidade ${ }^{7} 0$ recorte mais adequado ao 
exame das desigualdades sociais parece relacionarse com a identificação de situações que envolvem algum grau de injustiça com determinados coletivos populacionais - como os usuários visitados durante a realização deste estudo. 0 conceito de iniquidade ganhou forma e firmou-se como sinônimo de toda e qualquer "desigualdade injusta" a ser evitada ou combatida ${ }^{8,9}$.

0 cuidado domiciliar no âmbito da ESF é mais frequente no processo de trabalho de enfermeiros, médicos e técnicos/auxiliares de enfermagem em comparação ao trabalho de cirurgiões-dentistas e técnicos/auxiliares de saúde bucal, possivelmente em virtude de fatores como a inserção tardia da ESB na ESF e a dependência estrutural de tecnologias duras, associadas ao paradigma biomédico, ainda predominante na formação em Odontologia, que resulta em dificuldade de trabalhar em equipe e de lidar com ações coletivas ${ }^{13,14}$, mesmo que as diretrizes da PNSB, de 2004, estabeleçam a reorientação do modelo assistencial, com a ampliação do acesso à saúde bucal e a oferta de cuidado em todos os níveis de atenção à saúde ${ }^{15,16}$.

Vale ressaltar que, neste estudo, o êxito das VDs foi viabilizado pela mediação dos ACS, que favorece a execução das intervenções, as articulações locais e, em especial, o vínculo entre o CSF e o território, entre os serviços de saúde e a comunidade. A mediação dos ACS potencializa o acesso dos usuários, aumenta sua compreensão dos serviços e identifica as demandas de saúde, possibilitando um ajuste adequado entre os profissionais da saúde e a comunidade ${ }^{17}$.

Um importante achado da experiência aqui relatada foi a autopercepção negativa evidenciada por pacientes e cuidadores em termos de saúde bucal. Apesar de dores e infecções bucais terem o potencial de agravar condições sistêmicas, infelizmente, a saúde bucal ainda é considerada de baixa prioridade em comparação aos demais cuidados médicos voltados a esses usuários ${ }^{9}$. A vivência sinalizou que a maioria dos pacientes restritos ao lar não veem importância nos cuidados de saúde bucal. Mostrase indispensável difundir melhor as atribuições do cirurgião-dentista como profissional de saúde e não só como alguém que "cuida dos dentes"; seu paciente recebe uma assistência integral que melhora sua saúde como um todo.

Nas atividades formativas que têm os serviços de saúde e a comunidade como espaços de ensinoaprendizagem, é fundamental que professores e alunos aprofundem a humanização da atenção à

\section{...a saúde bucal ainda é considerada de baixa prioridade em comparação aos demais cuidados...}

saúde e elaborem estratégias para que o atendimento proposto pelo curso de Graduação em Odontologia possa dar respostas efetivas à comunidade, uma conquista ainda distante da realidade da própria ESF em grande parte dos municípios brasileiros ${ }^{13}$.

Por se tratar de um relato de experiência, este artigo apresenta a limitação de não fornecer resultados do uso do protocolo em questão a longo prazo, visto que o período de intervenção foi de um semestre. No entanto, o instrumento foi bem aceito pela equipe de saúde e pode ser monitorado em outras vivências no âmbito dos cursos de Graduação em Odontologia. Outra limitação se refere à não visitação de todos os pacientes restritos ao lar durante a intervenção, em virtude da alta demanda de trabalho dos profissionais do CSF. Isso torna necessário recorrer a um acompanhamento posterior para avaliar a exequibilidade desse protocolo.

\section{CONCLUSÃO}

Constatou-se que a $A D$ em saúde bucal apresenta pontos positivos, pois leva assistência a uma parcela da população que não teria acesso a serviços de saúde, devido à sua condição de restrição ao lar. Por meio da VD, os membros da ESB podem refletir sobre seu processo de trabalho com vistas a desenvolver estratégias que viabilizem as ações de saúde na comunidade e reduzam as desigualdades de acesso aos serviços de saúde.

Os resultados do uso do protocolo apontaram o fortalecimento do vínculo entre a população e a ESB. A inserção da AD nas atividades da ESB se encontra em evolução e é importante ressaltar que tais ações devem inserir-se no processo de trabalho para alcançar um vínculo mais efetivo entre a ESB e a comunidade assistida, viabilizando uma maior humanização da atenção à saúde e dos cuidados em saúde bucal.

Essa integração ensino-serviço-comunidade se 
mostra crucial para aumentar o número de visitas domiciliares a pacientes restritos ao lar no território, mediante ações de prevenção de agravos e promoção da saúde que facilitem a elaboração de um plano de cuidado para cada paciente. A longo prazo, esperase obter a continuidade das visitas a cada paciente, intensificar as intervenções necessárias e diminuir a demanda de procedimentos curativistas no âmbito da ESF.

\section{CONTRIBUIÇÃO DOS AUTORES}

André Pereira de Lima e Timóteo Sousa Lopes contribuíram com a realização da pesquisa, o delineamento do estudo e a redação do manuscrito. Atanara Freires Aguiar de Lima contribuiu com a redação do manuscrito. Mariana Ramalho de Farias e Jacques Antonio Cavalcante Maciel contribuíram com a redação e revisão crítica do manuscrito.

\section{REFERÊNCIAS}

1. Barbosa DCM, Mattos ATR, Corrêa MH, Faria M, Ribeiro LC, Santos LL, et al. Visita domiciliar sob a percepção dos usuários da ESF. Medicina (Ribeirão Preto) [serial on the internet]. 2016 [cited 2019 May 27];49(4):360-6. Available from: http://revista. fmrp.usp.br/2016/vol49n4/DMT-Visita-domiciliarsob-a-percepcao-dos-usuarios-da-ESF.pdf

2. Cunha MS, Sá MC. A visita domiciliar na Estratégia de Saúde da Família: os desafios de se mover no território. Interface Comun Saúde Educ [serial on the internet]. 2013 [cited 2019 May 27];17(44):6173. Available from: http://www.scielo.br/pdf/icse/ v17n44/a06v17n44.pdf

3. Maciel JAC, Almeida AS, Menezes AKA, Oliveira Filho IL, Teixeira AKM, Castro-Silva II, et al. Quando a saúde bucal bate à porta: protocolo para a atenção domiciliar em odontologia. Rev Bras Promoç Saúde [serial on the internet]. 2016 [cited 2019 May 27];29(4):614-20. Available from: https:// periodicos.unifor.br/RBPS/article/view/5463/pdf

4. Nishide $R$, Mizutani $M$, Tanimura $S$, Kudo $\mathrm{N}$, Nishii T, Hatashita H. Homecare protective and risk factors for early childhood caries in Japan. Environ Health Prev Med. 2018;23(1):57.

5. De-Carli AD, Santos MLM, Souza AS, Kodjaoglanian VL, Batiston AP. Visita domiciliar e cuidado domiciliar na atenção básica: um olhar sobre a saúde bucal. Saúde Debate [serial on the internet]. 2015 [cited 2019 May 27];39(105):441-50. Available from: http://www.scielo.br/pdf/sdeb/v39n105/0103-1104sdeb-39-105-00441.pdf
6. Bizerril D0, Saldanha KGH, Silva JP, Almeida JRS, Almeida MEL. Papel do cirurgião-dentista nas visitas domiciliares: atenção em saúde bucal. Rev Bras Med Fam Comunidade [serial on the internet]. 2015 [cited 2019 May 27];10(37):1-8. Available from: https:// www.rbmfc.org.br/rbmfc/article/view/1020/732

7. Travassos C, Martins M. Uma revisão sobre os conceitos de acesso e utilização de serviços de saúde. Cad Saúde Pública [serial on the internet]. 2004 [cited 2018 Dec 27];20(Suppl 2):S190-S198. Available from: http://www.scielo.br/pdf/csp/ v20s2/14.pdf

8. Barros FPC, Sousa MF. Equidade: seus conceitos, significações e implicações para o SUS. Saúde Soc [serial on the internet]. 2016 [cited 2019 May 27];25(1):9-18. Available from: http:// www.scielo.br/pdf/sausoc/v25n1/1984-0470sausoc-25-01-00009.pdf

9. Ferraz GA; Leite ICG. Instrumentos de visita domiciliar: abordagem da odontologia na estratégia saúde da família. Rev APS [serial on the internet]. 2016 [cited 2019 May 27];19(2):302-14. Available from: file:///D:/15647-Texto $\% 20$ do $\% 20$ artig 0-67104-1-10-20170118.pdf

10. Cheng YM, Ping CC, Ho CS, Lan SJ, Hsieh YP. Home-care aides' self-perception of oral health-care provision competency for community-dwelling older people. Int Dent J. 2019;69(2):158-64.

11. Souza DMM. A prática diária na Estratégia Saúde da Família. Juiz de Fora (MG): Ed. UFJF; 2011.

12. Silva LWS, Araújo TC, Santos FF, Lima AA, Santos GB, Lima LV. A família na convibilidade com o idoso acamado no domicílio. Rev Kairós [serial on the internet]. 2011 [cited 2019 May 27];14(3):75-87. Available from: https://revistas.pucsp.br/kairos/ article/view/6488/4704

13. Noro LRA, Torquato SM. Visita domiciliar: estratégia de aproximação à realidade social? Trab Educ Saúde [serial on the internet]. 2015 [cited 2019 May 27];13(1):145-57. Available from: http:// www.scielo.br/pdf/tes/v13n1/1981-7746-tes-19817746-sip00027.pdf

14. Aquilante AG, Silva GGA. 0 cuidado em saúde bucal após a Política Nacional de Saúde Bucal - "Brasil Sorridente": um estudo de caso. Ciênc Saúde Colet [serial on the internet]. 2015 [cited 2019 May 27];20(1):239-48. Available from: http:// www.scielo.br/pdf/csc/v20n1/pt 1413-8123csc-20-01-00239.pdf

15. Brasil. Diretrizes da Política Nacional de Saúde Bucal. Brasília (DF): Ministério da Saúde; 2004. 
16. Mattos GCM, Ferreira EF, Leite ICG, Greco RM. A inclusão da equipe de saúde bucal na Saúde da Família: entraves, avanços e desafios. Ciênc Saúde Colet [serial on the internet]. 2014 [cited 2019 May 27];19(2):373-82. Available from: http://www.scielo. $\mathrm{br} / \mathrm{pdf} / \mathrm{csc} / \mathrm{v} 19 \mathrm{n} 2 / 1413-8123-\mathrm{csc}-19-02-00373 . \mathrm{pdf}$

17. Muniz EA, Freitas CASL, Albuquerque IMN, Linhares MSC. Assistência domiciliar ao idoso no contexto da Estratégia Saúde da Família: análise da produção científica. Sanare (Sobral, Online) [serial on the internet]. 2014 [cited 2019 May 27];13(2):8691. Available from: https://sanare.emnuvens.com. br/sanare/article/view/578/311

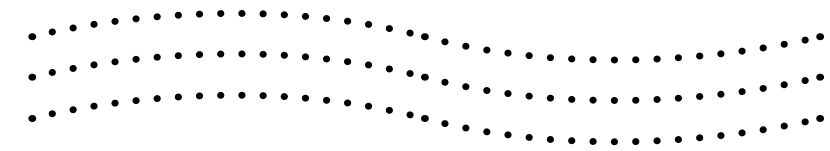

$\ldots \ldots \ldots \ldots \ldots \ldots \ldots \ldots \ldots \ldots \ldots \ldots \ldots \ldots \ldots \ldots \ldots \ldots$
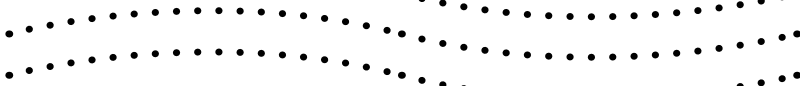
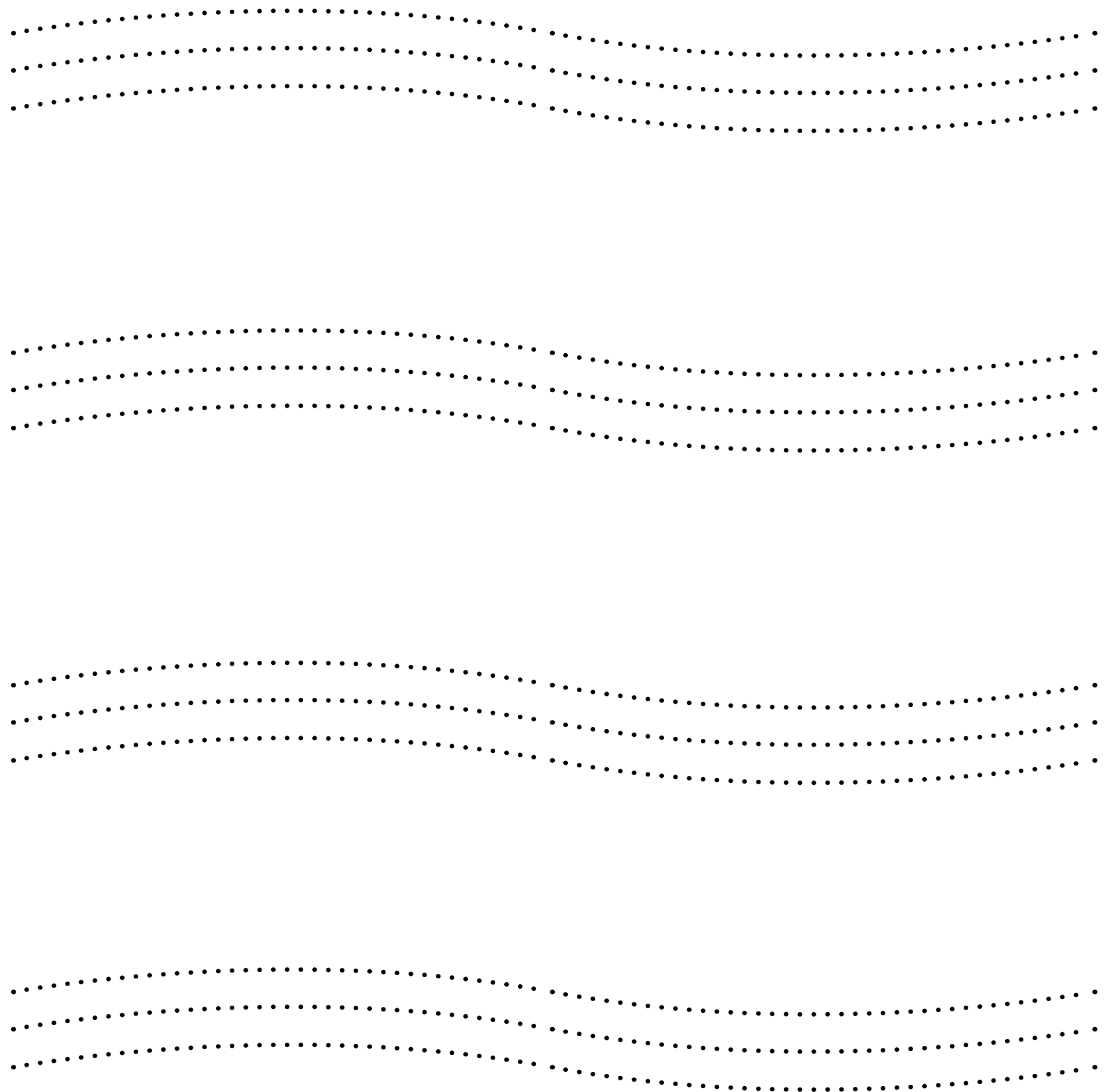